\title{
Circadian rhythm of biological processes in mouse liver and kidney: analysis of RNA-seq and ribosome profiling data
}

\author{
N.L. Podkolodnyy ${ }^{1,2 *}$, N.N. Tverdokhleb ${ }^{1,3}$, O.A. Podkolodnaya ${ }^{1}$ \\ ${ }^{1}$ Institute of Cytology and Genetics SB RAS, Novosibirsk, Russia \\ ${ }^{2}$ Institute of Computational Mathematics and Mathematical Geophysics SB RAS, Novosibirsk, Russia \\ ${ }^{3}$ Novosibirsk State University, Novosibirsk, Russia \\ *e-mail:pnl@bionet.nsc.ru
}

Key words: circadian rhythm, translation, GO enrichment analysis, tissue specificity, biological processes, phase characteristics

Motivation and Aim: A large-scale analysis of the circadian dynamics of transcriptomes and translatomes makes it possible to understand the role of transcriptional and posttranscriptional regulation of circadian clocks of the organism better and to reveal their relationship with the daily dynamics of biological processes. The purpose of this work is to study the tissue-specific features of the circadian phase characteristics of biological processes based on the GO functional analysis of genes with a pronounced circadian dynamics of transcription and translation in the mouse liver and kidney.

Methods and Algorithms: We used experimental data on the gene expression at the mRNA level and ribosome profiling (GSE67305 and GSE81283) in mouse liver and kidney. Identification of genes demonstrating the circadian dynamic is carried out by a modified method based on JTK_CYCLE. Identification of circadian patterns of gene expression (mRNA level and ribosome profiling) is carried out by the methods of correlation, cluster analysis and the principal component analysis. An analysis of the enrichment of groups of genes by the terms of GO was carried out using the bioinformatics resource DAVID. Results: We have identified genes that demonstrate pronounced circadian dynamics of transcription and translation. For the twelve time points (ZT0-ZT22) we have identified for each of the tissues groups of genes that were in a phase with an increased level of expression. For these groups of genes the GO enrichment analysis was carried out.

We have identified processes, the rhythmicity of which is characteristic for both the liver and the kidneys. Some of these processes showed similar circadian phase characteristics. At the same time, processes with significantly different temporal phase patterns in these two organs were identified. Also revealed processes with strict tissue-specific rhythmic translation. The circadian patterns of the mRNA level and ribosome profiling of the liver and kidney genes of the mouse were revealed. We analyzed the distribution of gene expression patterns across these 12 groups of genes and shown, in particular, that genes with a pronounced the impulse pattern of expression (the gene is expressed shortly during the day (no more than 2-4 hours)) most often have a maximum of expression at ZT16.

Conclusion: Our approach allows us to analyze the tissue-specific phase characteristics of biological processes, and the results emphasize the need to take into account the phase circadian characteristics when comparing the features of their course of these processes in various organs.

Acknowledgements: Supported by Presidium of SD RAS (0324-2018-0021) and by RF Government (0324-2018-0017). 